\title{
A changing picture of shigellosis in southern Vietnam: shifting species dominance, antimicrobial susceptibility and clinical presentation
}

\author{
Ha Vinh1,2, Nguyen Thi Khanh Nhu1,2, Tran Vu Thieu Nga1,2, \\ Pham Thanh Duy ${ }^{1,2}$, James I Campbell ${ }^{2,3}$, Nguyen Van Minh Hoang1,2, \\ Maciej F Boni ${ }^{2,3,4}$, Phan Vu Tra My ${ }^{1,2}$, Christopher Parry 2 , Tran Thi \\ Thu Nga1,2, Pham Van Minh1,2, Cao Thu Thuy ${ }^{1,2}$, To Song Diep², \\ Le Thi Phuong5, Mai Thu Chinh², Ha Thi Loan², Nguyen Thi Hong Tham5, \\ Mai Ngoc Lanh², Bui Li Mong5, Vo Thi Cuc Anh ${ }^{5}$, Phan Van Be Bay, \\ Nguyen Van Vinh Chau ${ }^{2}$, Jeremy Farrar $^{1,2}$ and Stephen Baker*1,2
}

Address: ${ }^{1}$ The Hospital for Tropical Diseases, Ho Chi Minh City, Vietnam, ${ }^{2}$ Oxford University Clinical Research Unit, Hospital for Tropical Diseases, Ho Chi Minh City, Vietnam, ${ }^{3}$ Centre for Tropical Medicine, Nuffield Department of Clinical Medicine, Oxford University, Oxford, UK, ${ }^{4}$ The MRC Centre for Genomics and Global Health, Oxford, UK and ${ }^{5}$ Dong Thap Provincial Hospital, Dong Thap, Vietnam

Email: Ha Vinh - vinhh@oucru.org; Nguyen Thi Khanh Nhu - nhuntk@oucru.org; Tran Vu Thieu Nga - ngatvt@oucru.org; Pham Thanh Duy - duypt@oucru.org; James I Campbell - jcampbell@oucru.org; Nguyen Van Minh Hoang - hoangnvm@oucru.org; Maciej F Boni - mboni@oucru.org; PhanVu Tra My - mypvt@oucru.org; Christopher Parry - cmparry@liverpool.ac.uk; Tran Thi Thu Nga - ngattt@oucru.org; Pham Van Minh - minhpv@oucru.org; Cao Thu Thuy - thuyct@oucru.org; To Song Diep - tsdiep@oucru.org; Le Thi Phuong - ltph@ourcu.org; Mai Thu Chinh - MTM@oucru.org; Ha Thi Loan - LTHA@oucru.org; Nguyen Thi Hong Tham - THAMTHN@oucru.org; Mai Ngoc Lanh - mainl@oucru.org; Bui Li Mong - monglbui@oucru.org; Vo Thi Cuc Anh - anhtcv@oucru.org; Phan Van Be Bay - bayvbp@oucru.org; Nguyen Van Vinh Chau - chaunvv@oucru.org; Jeremy Farrar - jfarrar@oucru.org; Stephen Baker* - sbaker@oucru.org

* Corresponding author

Published: 15 December 2009

BMC Infectious Diseases 2009, 9:204

This article is available from: http://www.biomedcentral.com/I47I-2334/9/204

(c) 2009 Vinh et al; licensee BioMed Central Ltd.

This is an Open Access article distributed under the terms of the Creative Commons Attribution License (http://creativecommons.org/licenses/by/2.0), which permits unrestricted use, distribution, and reproduction in any medium, provided the original work is properly cited.

\begin{abstract}
Background: Shigellosis remains considerable public health problem in some developing countries. The nature of Shigellae suggests that they are highly adaptable when placed under selective pressure in a human population. This is demonstrated by variation and fluctuations in serotypes and antimicrobial resistance profile of organisms circulating in differing setting in endemic locations. Antimicrobial resistance in the genus Shigella is a constant threat, with reports of organisms in Asia being resistant to multiple antimicrobials and new generation therapies.
\end{abstract}

Methods: Here we compare microbiological, clinical and epidemiological data from patients with shigellosis over three different periods in southern Vietnam spanningl 4 years.

Results: Our data demonstrates a shift in dominant infecting species (S. flexneri to S. sonnei) and resistance profile of the organisms circulating in southern Vietnam. We find that there was no 
significant variation in the syndromes associated with either S. sonnei or S. flexneri, yet the clinical features of the disease are more severe in later observations.

Conclusions: Our findings show a change in clinical presentation of shigellosis in this setting, as the disease may be now more pronounced, this is concurrent with a change in antimicrobial resistance profile. These data highlight the socio-economic development of southern Vietnam and should guide future vaccine development and deployment strategies.

Trial Registration: Current Controlled Trials ISRCTN5594588 I

\section{Background}

Shigellosis is an ongoing global public health problem. Due to the fecal-oral transmission route of the organisms, the overwhelming burden of shigellosis is found in resource-poor settings with inadequate sanitation $[1,2]$. With an estimated number of episodes exceeding 90 million per annum in Asia alone, shigellosis represents a significant proportion of the total number of bacterial gastrointestinal infections worldwide [3]. Unlike other related bacteria which can cause a particular disease syndrome in specific locations (e.g. Salmonella Typhi) [4] it is a disease which "bridges the gap" between industrialized and developing countries. A report from the National Center for Infectious Diseases in the United States of America found the incidence of shigellosis to be 7.6 cases per 100,000 persons in 1993 [5].

The Shigellae are gram negative, non-motile bacilli of the larger bacterial family Enterobacteriaceae. S. flexneri are regarded to be the most abundant globally and are known to predominate in developing countries [3]. S. sonnei is the most commonly isolated species in developed countries, representing over $70 \%$ of the total isolates in the United States of America and Israel [5,6]. The disease syndrome associated with these organisms includes fever, headache, malaise, anorexia and occasionally vomiting, followed by excretion of profuse watery diarrhea proceeding bloody and/or mucoid diarrhea [7]. All the members of the genus Shigella are pathogens restricted to infecting humans and exert their effects on the gastrointestinal mucosa via the production of a multitude of virulence factors, including enterotoxins and effector proteins $[8,9]$.

In a recent publication by von Seidlein et al. the authors found a change in dominant Shigella species related to the location in Asia (S. sonnei predominated in Thailand, $S$. flexneri was dominant in other Asian countries) and fluctuations in $S$. flexneri serotypes in the same location over the duration of the study [10]. The authors concluded that "Shigella appears to be more ubiquitous in Asian impoverished populations than previously thought and antibiotic-resistant strains of different species and serotypes have emerged" [10]. Such findings have important implications for treatment and prevention strategies of shigellosis.
On a larger scale, the Shigellae are a group of dynamic organisms, in which the overall bacterial population appears to be adaptable with a high recombination rate and a large amount of imported genetic material in the genome architecture [11]. These organisms are highly promiscuous regarding their ability to accept horizontally transferred genetic material. Like E. coli the Shigellae are successful recipients of numerous plasmids, which may be transferred from other enteric organisms in the gastrointestinal tract [12]. This is supported by evolutionary evidence that the Shigellae are a branch of the E. coli family, having developed a pathogenic phenotype by the acquisition of a virulence plasmid and other gene loci and genomic compensatory mechanisms $[13,14]$.

It is known that the circulating species and serotypes may be considered a marker of the socio-economic climate in an individual setting [15]. It is clear that Vietnam has undergone rapid economic development since the early 1990 's. To understand the nature of bacterial and clinical nature of shigellosis in southern Vietnam we have amassed and compared microbiological and epidemiological data on childhood shigellosis over three periods spanning 14 years, from 1995 to 2008.

\section{Methods \\ Study sites and settings}

The primary location was the pediatric gastrointestinal infections ward at the hospital for tropical diseases (HTD) in Ho Chi Minh City in southern Vietnam. The HTD is a 500 bed tertiary referral hospital treating patients from the surrounding provinces and from the districts within Ho Chi Minh City. The secondary location was Dong Thap provincial (DTP) hospital in Dong Thap province, approximately $120 \mathrm{~km}$ from the HTD in Ho Chi Minh City.

\section{Studies contributing data for analysis}

Data from three independent studies were combined and compared. All patients enrolled in the three studies were treated as inpatients and there were no fatalities. The initial period (referred to as period A from here onwards) was a study performed at the pediatric ward at HTD from January 1995 to August 1996. The enrollment and clinical observations for this randomized controlled trial are as 
described previously [16]. Briefly, children that were aged $>3$ months and $<14$ years, admitted to HTD with fever and bloody diarrhea (bloody diarrhea defined $>3$ loose stools with obvious blood) for $<5$ days were entered into the study provided that their parents or guardian gave fully informed consent. Additional strains for microbiological assessment only (nine in total) were collected for comparison within the same period of the study duration from DTP. Overall 80 strains were isolated from enrolled children over this period; clinical data was available for analysis on 63 patients with culture confirmed shigellosis.

The secondary period (referred to as period $\mathrm{B}$ from here onwards) was conducted only at the HTD, between March 2000 and December 2002. This period was a clinical and microbiological investigation of the etiology of diarrhea in the pediatric population admitted to the HTD in Ho Chi Minh City. Whilst the treatment criteria for this descriptive study were not controlled ( $>90 \%$ of patients received treatment with fluroquinolones (norfloxacin or ofloxacin)), the remainder of the criteria for admission to the study were comparable, children were eligible for enrollment to the study if consent was given and they were aged less than 14 years. The obvious variation in the enrollment for this study was that children were enrolled on the basis of having any diarrheal syndrome, rather than specifically targeting those with dysentery and suspected shigellosis. One hundred and fourteen Shigella isolates were recovered during this period; clinical data was available for analysis on 113 patients.

The final period (referred to as period $\mathrm{C}$ from here onwards) in which data was combined was a trial conducted at the HTD and at DTP between June 2006 and December 2008. This was a randomized controlled trial for comparing the treatment of dysentery with ciprofloxacin and gatifloxacin in Vietnamese children (controlled trials number ISRCTN55945881) (HV and SB, unpublished data). The inclusion criteria were as period A. One hundred and three isolates were collected during this period and clinical data on all admitted children was available for analysis.

All three studies were approved ethical assessment by the Scientific and Ethical Committee of the hospital for tropical diseases and Oxford University tropical ethics committee (OXTREC) number 010-06 (2006).

\section{Microbiological methods}

From all studies, stool samples were collected from patients and cultured directly on the day of sampling. Initial isolation was as below, however, all bacterial isolates were stored in glycerol at $-80^{\circ} \mathrm{C}$ and re-serotyped and checked for consistency with the original antimicrobial susceptibility profile for the purposes of this work. All specimens were processed and checked in the microbiology laboratory of the HTD.

Samples were cultured overnight in selenite $\mathrm{F}$ broth (Oxoid, Basingstoke, UK) and onto MacConkey and XLD agar (Oxoid) at $37^{\circ} \mathrm{C}$. Colonies suggestive of Salmonella or Shigella (non-lactose fermenting) were sub-cultured on to nutrient agar and were identified using a 'short set' of sugar fermentation reactions (Kliger iron agar, urea agar, citrate agar, SIM motility-indole media (Oxoid)). After incubation for $18-24 \mathrm{~h}$ at $37^{\circ} \mathrm{C}$, the test media were read for characteristic Shigella reactions and API 20E test strips of biochemical reactions (Biomerieux, Paris, France) were used to confirm the identity of Shigella spp. Serologic identification was performed by slide agglutination with polyvalent somatic $(\mathrm{O})$ antigen grouping sera, followed by testing with available monovalent antisera for specific serotype identification as per the manufacturers recommendations (Denka Seiken, Japan).

Antimicrobial susceptibility testing of all Shigella isolates against ampicillin (AMP), chloramphenicol (CHL), trimethoprim- sulfamethoxazole (SXT), tetracycline (TET), nalidixic acid (NAL), ofloxacin (OFX) and ceftriaxone (CRO) was performed by disk diffusion following standardized Clinical and Laboratory Standards Institute methods [17]. The minimum inhibitory concentrations (MICs) were additionally calculated for all isolates by E-test, according to manufacturer's recommendations (AB Biodisk, Solna, Sweden) and were compared to control strain E. coli ATCC 25922 and an in house fully sensitive E. coli control.

\section{Clinical observations and statistical analysis}

Clinical data was recorded on specialized clinical report forms for all three studies by clinical staff involved in the studies. The data collected was related to basic details of the patient, age (months), sex, location of residence and weight (kg). A history from all patients was also recorded, including; duration of illness prior to admission to hospital (days), fever (defined as a prolonged temperature > $\left.37.5^{\circ} \mathrm{C}\right)$, abdominal discomfort, vomiting, watery diarrhea (defined as three or more loose bowel movements during a 24-h period), bloody or mucoid diarrhea (defined as $>3$ loose stools with obvious blood or mucus), estimated number of episodes of diarrhea before attending hospital, convulsions believed to be related to fever and/or infection and if there was any known pretreatment with antimicrobials. A white blood cell count was performed on all patients and stools were examined by microscopy (HPF $(\times 400))$ to identify white and red blood cells, these observations were scored on scale from zero to three, scale $0=0$ cells $/ \mathrm{HPF}$, scale $1=1$ to 10 cells $/ \mathrm{HPF}$, scale $2=11$ to 20 cells $/ \mathrm{HPF}$ and scale $3=>20$ cells/HPF. Time in hours (from initial investigation in hospital) to 
the ceasing of bloody/mucoid and watery diarrhea was recorded. Duration of hospital stay was recorded in days post admission; patients were only discharged when all clinical symptoms had resolved completely.

Data were double entered into Microsoft Excel for storage and manipulation. Mapping data was entered, analyzed and draw in MapInfo software (Pitney Bowes MapInfo Corporation, USA). For intergroup comparisons, Chisquare tests were used for comparison of categorical variables. For the analysis of continuous variables, Wilcoxon rank sum, and Kruskal-Wallis test were used for non-nor- mally distributed data. A p-value of less than 0.05 (twotailed) was considered significant. Statistical analysis was performed in R http://www.r-project.org/.

\section{Results \\ Epidemiological findings}

Over the duration of the three periods spanning 14 years, 228 Shigellae were isolated from children living within 13 districts that constitute Ho Chi Minh City (Figure 1). Whilst the distribution of the location of the residences of these patients is biased by referral patterns and people attending the local hospital (HTD is one of several hospi-

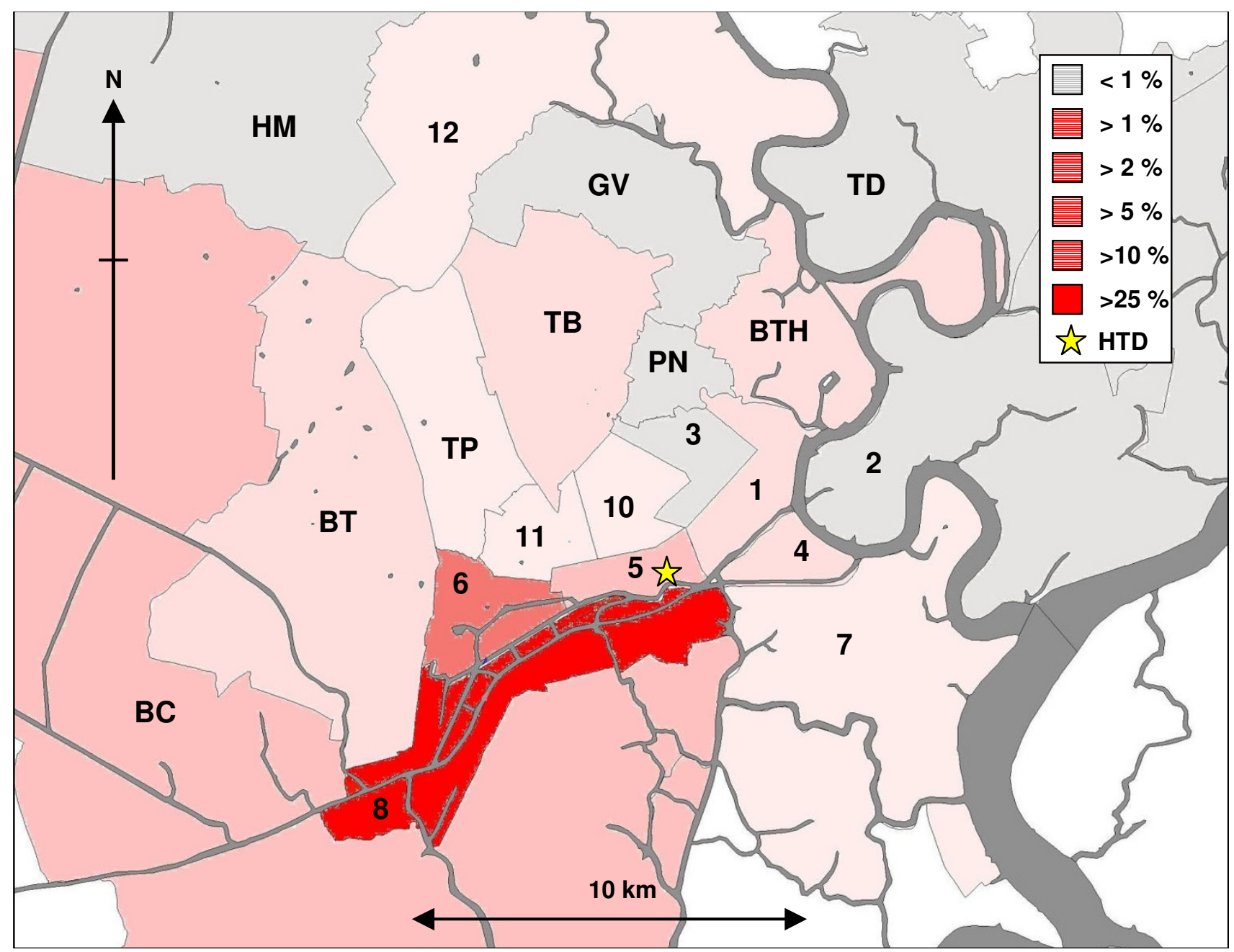

\section{Figure I}

The distribution of the residences of cases of childhood shigellosis admitted to the hospital for tropical diseases in Ho Chi Minh City. Over the three periods we were able to positively identify infecting Shigella serotypes in the stools of 297 children with symptoms consistent with shigellosis. Of these patients, 228 (76.8\%) children lived in the 23 districts that constitute Ho Chi Minh City. This figure represents the distribution of the homes of patients reporting to the hospital for tropical diseases with Shigella isolated from stool, by district. The percentage of cases reporting from each ward is distinguished by gradual shading. The location of the hospital for tropical diseases is shown by a yellow star. Large waterways (rivers and canals) are shown in dark grey shading. 
tals in the City where children may be treated for gastrointestinal infections), the majority of children attending HTD with culture confirmed shigellosis came from the three districts within the locality of the hospital (districts 5,6 and 8), which constitutes a total population of over 800,000 people. In total, the majority of the patients resided in district eight $(n=88)$ within approximately 6 $\mathrm{km}$ of the hospital. There was no significant change in the locality of patients over the three periods, or any relationship between serotype and location of the residence of the patients.

The median age of children with culture confirmed shigellosis from all the combined data was 24 months; the age range was from 3 to 154 months (Figure 2). The number of children requiring hospital treatment as inpatients for shigellosis declined significantly after 36 months of age. The combined data from periods $\mathrm{A}, \mathrm{B}$ and $\mathrm{C}$ demonstrated some seasonality related to the times of peak infection, with the majority of cases (> 60\%) occurring in the wet season (between May and September) (Figure 3).

\section{Microbiology and antimicrobial susceptibilities}

In total, 297 Shigella strains were isolated from periods A, B and C. Three were S. boydii, 136 were S. flexneri, 149 were S. sonnei and nine were untypeable. There was a significant species shift from $S$. flexneri to $S$. sonnei between period A (29\% S. sonnei) and period C (78\% S. sonnei) with an approximate $1: 1$ ratio of $S$. flexneri to $S$. sonnei in the intermediate period (Figure 4). Apart from $S$. flexneri serotype one only being found in period $A$, there was no evident fluctuations in $S$. flexneri populations between the three periods. The most commonly isolated $S$. flexneri serotype was serotype $2 \mathrm{a}$; representing $43 \%$ of all the $S$. flexneri strains (Table 1).

We identified a significant change in the profile of the proportions of organisms demonstrating resistance to seven antimicrobials (Figure 5). There was a sequential increase in the number of Shigellae isolated that were resistant to nalidixic acid, ofloxacin and ceftriaxone. In period C, 23\% of strains were resistant to ceftriaxone and $68 \%$ were resistant to nalidixic acid (Figure 5). There was an additional overall increase in the number of antimicrobials to which the organisms were resistant. During period A, 62\% of all Shigellae were resistant to three or more of the seven antimicrobials tested, this increased to $87 \%$ in period $\mathrm{B}$ and decreased to $83 \%$ in period $\mathrm{C}$ (Figure 5 ). The proportion of organisms that were resistant to trimethoprim- sulfamethoxazole and tetracycline was unchanged between

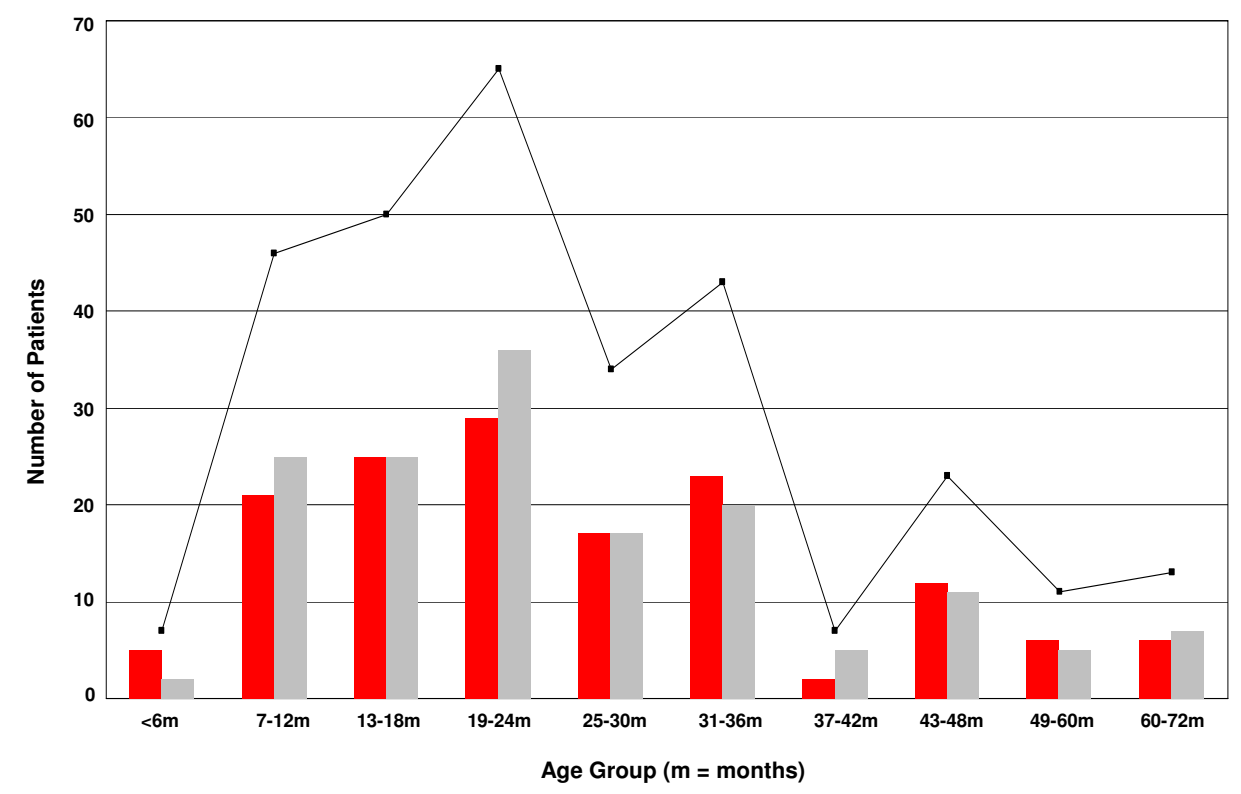

Figure 2

The combined sex and age distribution of childhood shigellosis patients in southern Vietnam. Graph depicts the combined age and sex distribution (female - red, male - grey) of 297 children with shigellosis. The black line with boxes represents the total number of cases per age group specified. The overall age range was from 3 months to I54 months, with a median of 24 months. There was no significant relationship of shigellosis with gender; in total, I52 patients were male (5I.2\%) and 145 were female $(48.8 \%)$. 


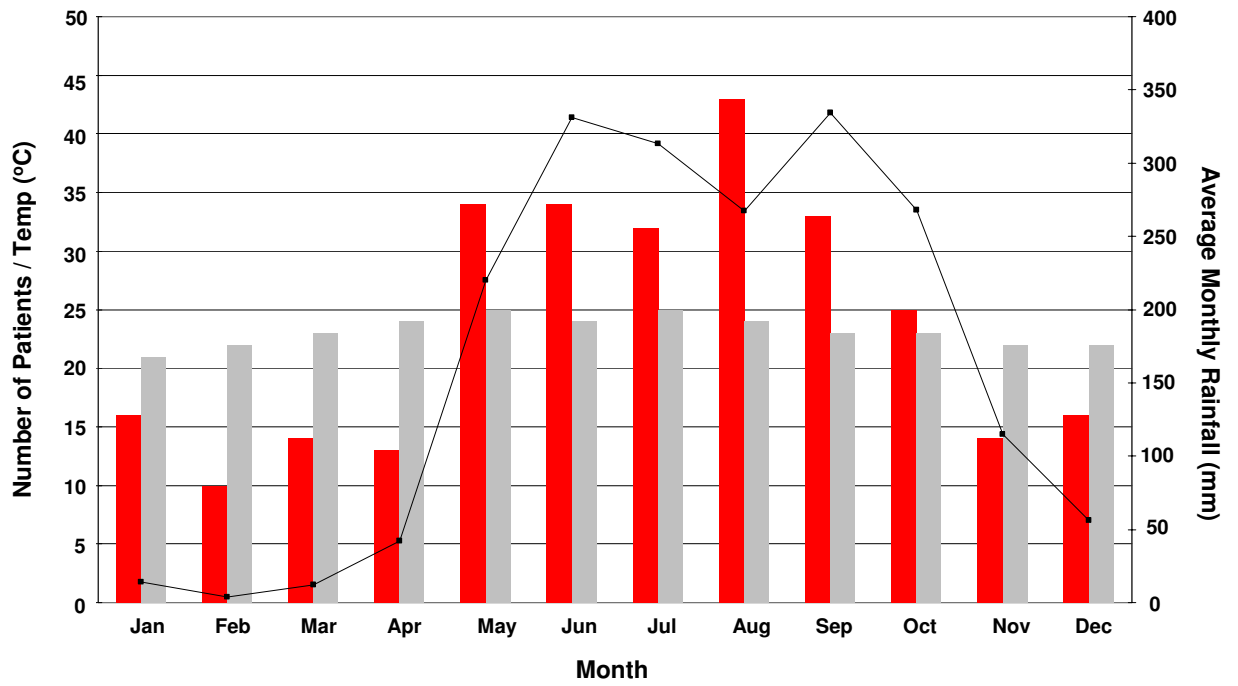

\section{Figure 3}

The seasonal distribution of shigellosis in southern Vietnam. southern Vietnam has two distinct seasons, wet and dry. The combined data were averaged by calculating the number of months represented to get an overall number of cases per month. Red bars; total number of cases, grey bars; average monthly temperature and black line with boxes; average monthly rainfall. The seasonal data represents the average rainfall and temperature per month for Ho Chi Minh City.

the three periods (Figure 5). Between period A and $\mathrm{C}$, there were significant decreases in the proportions of organisms resistant to ampicillin, decreasing from $75 \%$ to $48 \%$, and chloramphenicol, decreasing from $66 \%$ to $30 \%$.

There was a discernible change in sensitivity patterns over time, which was also related to Shigella species (Table 2). S. flexneri was significantly more likely to be resistant to ampicillin in periods $\mathrm{A}$ and $\mathrm{C}$ and when combined over all three studies. $S$. flexneri was also significantly more likely to be resistant to chloramphenicol in periods $\mathrm{B}, \mathrm{C}$ and overall (Table 2). The combined data demonstrated that $S$. sonnei was significantly more likely to be resistant to trimethoprim- sulfamethoxazole and ceftriaxone, despite ceftriaxone resistance not becoming evident till period $\mathrm{C}$. The overall pattern of reversion of sensitivity to ampicillin and chloramphenicol was mainly observed with respect to $S$. sonnei isolates. An increase in the number of organisms resistant to multiple antimicrobials over time was seen in both Shigella species. However, between period A and period C, S. flexneri was more likely to be resistant to more antimicrobials than $S$. sonnei (Figure 6). Resistance to multiple antimicrobials increased from two to three out of the seven tested from periods A to $\mathrm{C}$ for $\mathrm{S}$. sonnei and from four to five from the seven antimicrobials tested from periods A to C for S. flexneri (Figure $6)$.

\section{Clinical features associated with shigellosis}

Clinical data was combined and analyzed from all three studies; this permitted a comparison of some of the features of the patients with confirmed shigellosis over the three studies. Data were available for analysis from 279 patients; 63 patients from period A, 113 patients from

Table I: Shigella flexneri serotypes isolated in southern Vietnam between 1995 and 2008.

\begin{tabular}{ccc}
\hline S. flexneri serotype & Number & Percentage (\%) \\
\hline Ia & 0 & 0 \\
Ib & 0 & 0 \\
Ic & 4 & 2.9 \\
$2 \mathrm{a}$ & 59 & 43.4 \\
$2 \mathrm{~b}$ & 8 & 5.9 \\
$3 \mathrm{a}$ & 13 & 9.6 \\
$3 \mathrm{~b}$ & 2 & 1.5 \\
$3 \mathrm{c}$ & 16 & 11.8 \\
4 & 7 & 5.1 \\
$4 \mathrm{a}$ & 5 & 3.7 \\
$4 \mathrm{~b}$ & 1 & 0.7 \\
$4 \mathrm{x}$ & 0 & 0 \\
$5 \mathrm{a}$ & 0 & 0 \\
6 & 13 & 9.6 \\
$\mathrm{x}$ & 0 & 0 \\
$\mathrm{y}$ & 0 & 0 \\
Not typed & 8 & 5.9 \\
\hline Total & 136 & 100 \\
\hline
\end{tabular}




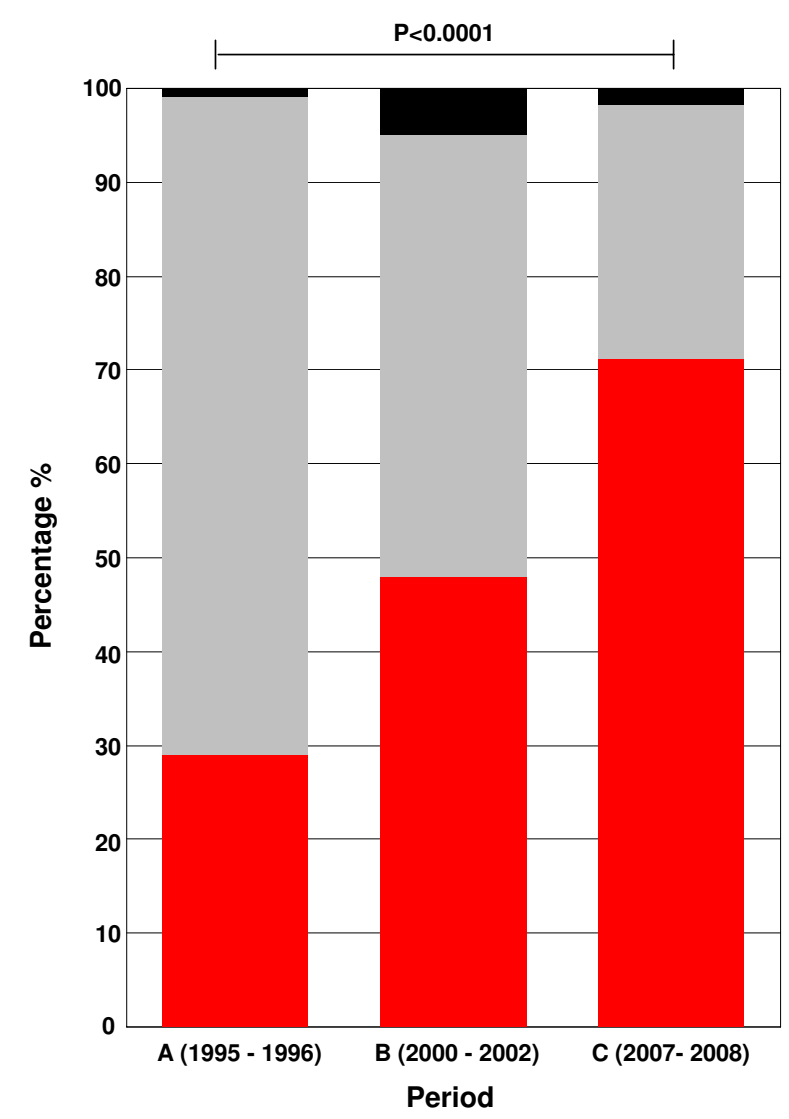

\section{Figure 4}

The distribution of Shigella species from three childhood shigellosis studies in southern Vietnam over fourteen years. The distribution of Shigella species from period $A(n=80)$, period $B(n=114)$ and period $C(n=103)$. The percentage of $S$. sonnei and $S$. flexneri are colored red and grey respectively, other Shigella species are colored black. The $p$ value was calculated using the chi - squared test.

period $\mathrm{B}$ and 103 patients from period $\mathrm{C}$ (Table 3). These data demonstrated several changes in disease profile over the three periods. There was a statistically significant increase in age, which corresponded with an increase in weight of the children from period A to period C (Table 3 ). There was decrease in the number of days of history of the disease symptoms prior to admission to hospital. There was a statistically significant increase in the number of children with watery diarrhea, abdominal pain and febrile convulsions. These clinical features combined suggested a progressively more severe infection syndrome between 1995 and 2008. Additionally, patients in period $\mathrm{C}$, had higher white blood cell counts. Over the 14 year period, patients had a higher density of white cells in their stool and had longer stays in hospital.
The increase in the severity of the disease was concurrent with a change in antimicrobial resistance profiles of the organisms and a change in the dominant Shigella species isolated. Therefore, these data suggested a more severe disease pattern may be related to infection with $S$. sonnei. To account for any variation in disease syndrome that may be species specific, the data were analyzed to compare the clinical syndromes related to species. The data presented in Table 4 demonstrates only subtle differences between the syndromes synonymous with the two differing species. S. flexneri shows an increase in the number of days of illness prior to admission in hospital, the number of episodes of diarrhea, an increase in the duration of mucoid/ bloody diarrhea and the duration of stay in hospital.

\section{Discussion}

Our findings demonstrate that the epidemiology of shigellosis infection is similar in southern Vietnam to other locations in Asia. The main burden of infection in children is in those under three years of age $[10,15,18,19]$. The median age of patients in this investigation was 24 months, this is slightly less than a previous study in Nha Trang, Central Vietnam [10]. A discrepancy in age in the two settings may be related to the epidemiological study being performed with ongoing community surveillance, rather than those admitted to hospital for treatment. We also found a pattern of infection which correlated with the rainy season. The observation that Shigella infections generally coincide with the wet season in a tropical setting has been noted before in an urban setting in Jakarta, Indonesia [18]. Transmission of Shigella has been associated with wastewater and river water in Vietnam in two independent locations in Vietnam [20,21]. An increase in fecal contamination of the water supply due to increased ground water may account for this pattern as distance to a water source was found to be associated with higher risk of shigellosis in Nha Trang. The majority of patients enrolled in the studies combined here resided in District 8 of Ho Chi Minh City. Although we are unable to draw meaningful conclusions from the residences of these patients owning to referral and catchment areas of the HTD, district 8 represents the area of the city with the greatest density of canal networks and waterways.

In addition to a species shift over time, there was combined effect on antimicrobial resistance; there was a marked increase in resistance to ceftriaxone and nalidixic acid. We have previously reported an alarming increase in ceftriaxone resistant Shigellae in southern Vietnam [22]. Whilst nalidixic acid is no longer used therapeutically, resistance increases the MIC to fluoroquinolones, which are recommended for the treatment of Shigella infections [23]. Our theory that antimicrobial resistant organisms are under selective pressure in this population is sup- 


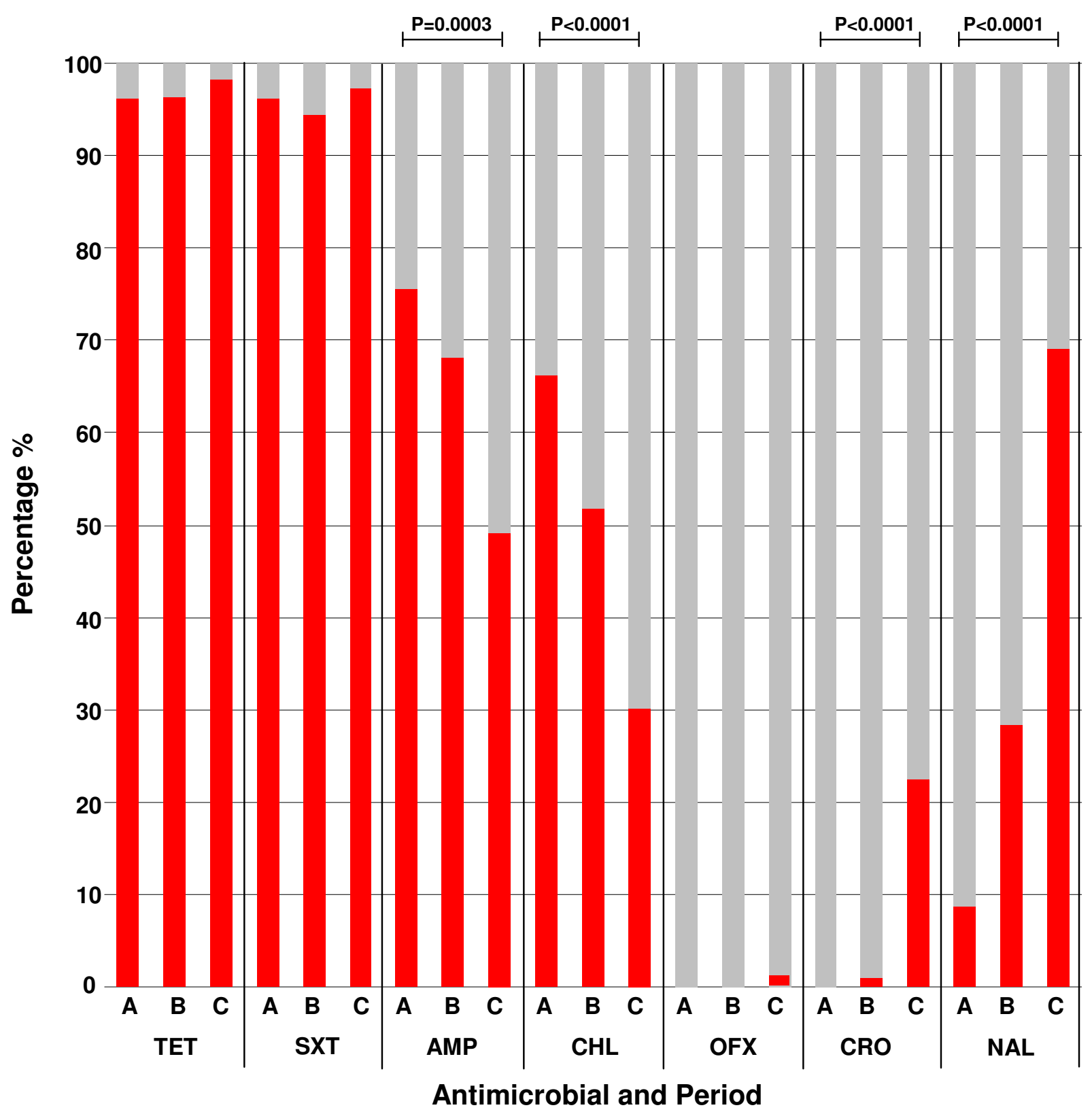

Figure 5

Changing antimicrobial resistance patterns of Shigella spp. All organisms were tested for susceptibility to seven antimicrobial agents by the disc diffusion and E-test methods. The antimicrobials tested were as follows, AMP; Ampicillin, CHL; Chloramphenicol, SXT; Trimethoprim- Sulfamethoxazole, TET; Tetracycline, NAL; Nalidixic Acid, OFX; Ofloxacin and CRO;

Ceftriaxone. Graph shows the percentage of resistant (red) and sensitive (grey) organisms isolated from periods A, B and C. Statistical significance was calculated using a chi squared test. 
Table 2: Comparison of resistance patterns between Shigella flexneri and Shigella sonnei isolated in southern Vietnam between 1995 and 2008.

\begin{tabular}{|c|c|c|c|c|c|c|c|c|c|}
\hline Collection & Serotype (n) & Phenotype $^{b}$ & AMP & $\mathrm{CHL}$ & SXT & TET & NAL & OFX & CRO \\
\hline \multirow[t]{4}{*}{ A (1995 - 1996) } & sonnei (24) & $\mathrm{R}$ & 11 & 8 & 23 & 23 & 0 & 0 & 0 \\
\hline & & s & 13 & 16 & 1 & 1 & 24 & 24 & 24 \\
\hline & flexneri (56) & $\mathrm{R}$ & 54 & 10 & 53 & 53 & 9 & 0 & 0 \\
\hline & & s & 2 & 46 & 3 & 3 & 47 & 56 & 56 \\
\hline$p$ Value ${ }^{a}$ & & & $<0.0001$ & 0.1287 & 0.8102 & 0.8102 & 0.0371 & - & - \\
\hline \multirow[t]{4}{*}{ B (2000 - 2002) } & sonnei (54) & $\mathrm{R}$ & 50 & 10 & 53 & 52 & 9 & 0 & 1 \\
\hline & & s & 4 & 44 & 1 & 2 & 45 & 54 & 53 \\
\hline & flexneri (50) & $\mathrm{R}$ & 46 & 38 & 44 & 49 & 21 & 0 & 0 \\
\hline & & S & 4 & 12 & 6 & 1 & 29 & 50 & 50 \\
\hline$p$ Value ${ }^{a}$ & & & 0.9316 & $<0.0001$ & 0.0415 & 0.5577 & 0.0052 & - & 0.329 \\
\hline \multirow[t]{4}{*}{$C(2007-2008)$} & sonnei (7I) & $\mathrm{R}$ & 17 & 5 & 71 & 69 & 51 & 0 & 12 \\
\hline & & s & 54 & 66 & 0 & 2 & 20 & 71 & 59 \\
\hline & flexneri (30) & $\mathrm{R}$ & 25 & 28 & 28 & 30 & 19 & 1 & 1 \\
\hline & & s & 5 & 2 & 2 & 0 & II & 29 & 29 \\
\hline$p$ Value $^{a}$ & & & $<0.0001$ & $<0.0001$ & $<0.0001$ & 0.3696 & 0.4619 & 0.297 & 0.076 \\
\hline \multirow[t]{4}{*}{ Combined } & sonnei (148) & $\mathrm{R}$ & 78 & 23 & 147 & 144 & 60 & 0 & 13 \\
\hline & & $\mathrm{s}$ & 70 & 125 & 1 & 4 & 88 & 148 & 135 \\
\hline & flexneri (136) & $\mathrm{R}$ & 125 & 76 & 125 & 132 & 49 & 1 & 1 \\
\hline & & s & 11 & 60 & 11 & 4 & 87 & 135 & 135 \\
\hline$p$ Value ${ }^{a}$ & & & $<0.0001$ & $<0.0001$ & 0.001 & 0.613 & 0.365 & 0.478 & 0.002 \\
\hline
\end{tabular}

a $p$ Value calculated using the chi-squared test

b Phenotype with respect to Resistant (R) or Sensitive (S)

AMP; Ampicillin, CHL; Chloramphenicol, SXT; Trimethoprim- Sulfamethoxazole, TET; Tetracycline, NAL; Nalidixic Acid, OFX; Ofloxacin, CRO; Ceftriaxone.

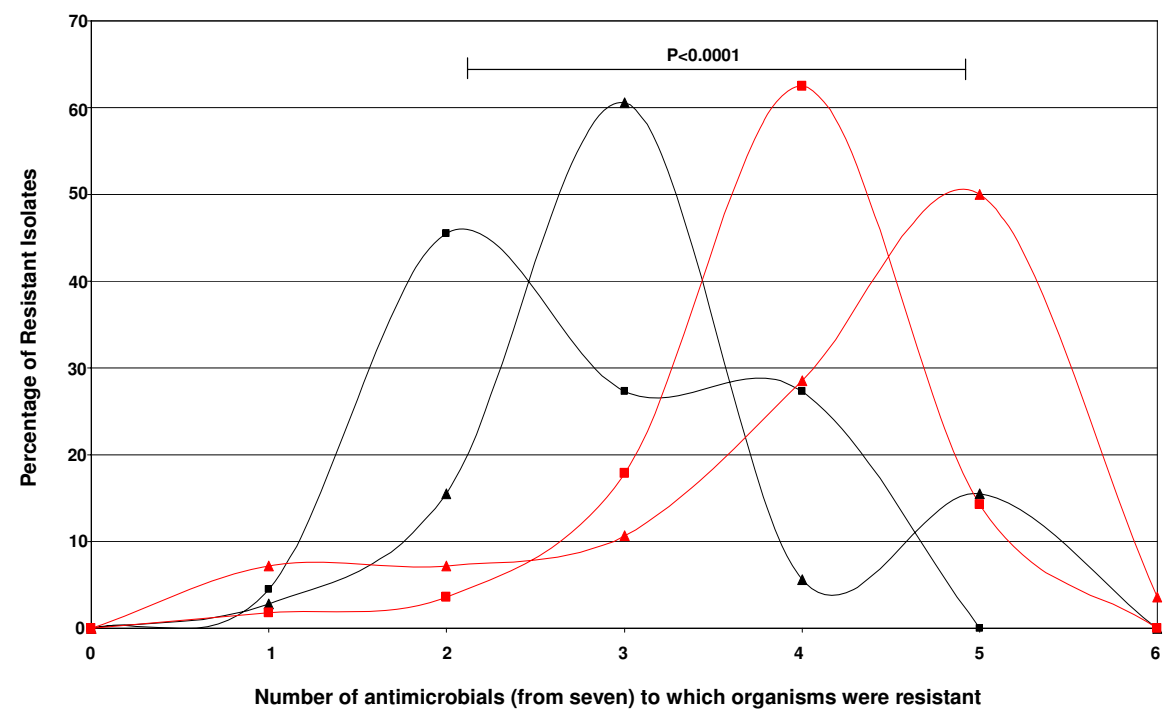

\section{Figure 6}

The increasing proportions of antimicrobial resistant S. sonnei and S. flexneri during a fourteen year transition. The distribution of the proportion of S. sonnei and S. flexneri isolates that were resistant to one or more of seven antimicrobials tested. S flexneri strains (red lines) were significantly more likely to be resistant to more antimicrobials that S. sonnei (black lines) over both collections compared. S. sonnei and S. flexneri were significantly more likely to be resistant to more antimicrobials when period C (2007 - 2008) (lines with triangles) was compared to period A (I995 - 1996) (lines with squares). 
Table 3: Clinical results of Shigella infections between 1995 and 2008.

\begin{tabular}{|c|c|c|c|c|c|}
\hline & $A(1995-1996)$ & B (2000 - 2002) & C (2007 - 2008) & Combined & $p$ value $^{a}$ \\
\hline Pateints & $n=63$ & $n=113$ & $n=103$ & $n=279$ & \\
\hline Age (months) ${ }^{d}$ & $23(17-48)$ & $21(14-29)$ & $30(19-42)$ & $24(16-36)$ & $<0.001$ \\
\hline Weight (kg) & $10(9-13)$ & $10(9-12)$ & $11.5(10-15)$ & $10.5(9-13)$ & 0.004 \\
\hline Male sex (\%) & $31(49)$ & $50(44)$ & $61(59)$ & $184(59 \%)$ & 0.085 \\
\hline \multicolumn{6}{|l|}{ Patient history } \\
\hline Days & $2(1-7)$ & $2(1-9)$ & I (I - 4) & $2(1-9)$ & $<0.001$ \\
\hline Fever $(\%)$ & $62(98)$ & $104(92)$ & $100(97)$ & $266(95)$ & 0.09 \\
\hline Abdominal pain (\%) & $33(52)$ & $41(36)$ & $79(76)$ & $153(54)$ & $<0.001$ \\
\hline Vomitting (\%) & $24(38)$ & $64(56)$ & $51(49)$ & $139(50)$ & 0.062 \\
\hline Watery diarrhea (\%) & $28(44)$ & $67(59)$ & $74(7 I)$ & $169(60)$ & 0.002 \\
\hline Bloody/mucoid diarrhea (\%) & $63(100)$ & $60(53)$ & $98(95)$ & $221(79)$ & $<0.001$ \\
\hline Diahorreal episodes per day & NA & $8(5-10)$ & $8(5-10)$ & $8(5-10)$ & 0.595 \\
\hline Convulsions (\%) & $4(6)$ & $7(6)$ & $20(19.4)$ & $31(I I)$ & $<0.001$ \\
\hline Known pretreatment (\%) & $3(5)$ & $8(7)$ & $4(4)$ & $14(5)$ & 0.543 \\
\hline \multicolumn{6}{|l|}{ Clinical details } \\
\hline Serotype sonnei (\%) & $21 / 63(33)$ & $55 / 113(49)$ & $71 / 103(69)$ & I53/279 (55) & $<0.001$ \\
\hline White cell count $\left(\times 10^{3} / \mathrm{mm}^{3}\right)$ & $10(8.3-15)$ & $10.1(7.7-12.8)$ & $13.1(10.1-17.3)$ & $11.3(8.7-15.4)$ & $<0.001$ \\
\hline Red cells in stoolb & NA & 1 & 1 & 1 & 0.715 \\
\hline White cells in stoolb & NA & 3 & 3 & 3 & 0.02 \\
\hline Mucoid duration (hrs) & $31.5(24-53.5)$ & $36(24-54)$ & $28(18-48)$ & $30(19.5-48)$ & 0.113 \\
\hline Diarrhea duration (hrs) & $48.5(29.25-87)$ & $48(24-72)$ & $48(30-72)$ & $48(26.75-72)$ & 0.402 \\
\hline \multicolumn{6}{|l|}{ Duration of illness } \\
\hline Hospital stay (days) & $3(1-12)$ & $4(1-15)$ & $5(2-14)$ & $4(1-15)$ & $<0.001$ \\
\hline Disease duration (days) ${ }^{c}$ & $4(2-15)$ & $6(3-18)$ & $6(3-15)$ & $6(2-18)$ & $<0.001$ \\
\hline
\end{tabular}

a $p$ Values calculated using either Chi-square test or the Kruskal-Wallis test

b Cells in Stools assessed as described in methods

c Disease duration calculated by addition of history of disease and stay in hospital

$d$ Interquartile range values in brackets unless stated

ported by a sequential decrease in resistance to older antimicrobial therapies, such as ampicillin and chloramphenicol which are now rarely used in the community to treat gastrointestinal infections. The uncontrolled use of antimicrobials in this setting may fuel the spread of multiple drug resistant organisms. However, due to promiscuous nature of the Shigellae it is likely that resistance genes are transferred regularly to and from other enteric bacteria and maintained by selective pressure. The change is species and antimicrobial resistance pattern reflects a change occurring in the Shigella population over time in this setting. Locality and time of isolation data suggest that entrance to all studies was sporadic and there was no evidence of transient epidemics.

Currently there are several candidate Shigella vaccines in development, of which some have already been tested in initial clinical trials [24-27]. The development and deployment of Shigella vaccines may be hindered by the number of different species and serotypes circulating in one setting and in differing locations. For example, $S$. flexneri serotypes are known to fluctuate over time, this has been observed in India, Indonesia, Bangladesh, and Pakistan, $[10,28]$. Here, we have demonstrated a significant longitudinal transition of species from S. flexneri to $S$. sonnei. Vaccine development for shigellosis is challenging as primary infection offers only serotype specific immunity [29]. A study concerning a cohort of Chilean children found infection conferred $76 \%$ protective efficacy against re-infection with the same serotype [30]. An option for controlling shigellosis would be the development of a series of single serotype vaccines which could be implemented in individual locations with a known serotype profile. Alternatively, the most cost effective method of control would be the development of a polyvalent vaccine offering cross protection to a number of known dominant 
Table 4: The clinical presentation of Shigella flexneri and Shigella sonnei infections.

\begin{tabular}{|c|c|c|c|}
\hline & S. flexneri & S. sonnei & $p$ value ${ }^{a}$ \\
\hline Pateints & $n=123$ & $n=147$ & \\
\hline Age (months) & $25(12-42)$ & $23(14-36)$ & 0.105 \\
\hline Weight (kg) & II (8.5- 14) & $10(9.9-13)$ & 0.558 \\
\hline Male sex $(\%)$ & $55(44.7)$ & $83(56.5)$ & 0.055 \\
\hline \multicolumn{4}{|l|}{ Patient history } \\
\hline Days & $2(2-3)$ & $I(I-2)$ & $<0.001$ \\
\hline Fever (\%) & $117(95)$ & $|4|(96)$ & 0.761 \\
\hline Abdominal pain (\%) & $64(52)$ & $84(57.1)$ & 0.48 \\
\hline Vomitting (\%) & $60(48.8)$ & $74(50.3)$ & 0.78 \\
\hline Watery diarrhea (\%) & $78(63.4)$ & $86(58.5)$ & 0.41 \\
\hline Bloody/mucoid diarrhea (\%) & $97(78.9)$ & $117(80)$ & 0.88 \\
\hline Diarrhea episodes per day & $10(5-10)$ & $8(5-10)$ & 0.051 \\
\hline Convulsions (\%) & $9(7.3)$ & $21(14.3)$ & 0.07 \\
\hline Known pretreatment (\%) & $7(5.7)$ & $7(4.8)$ & 0.585 \\
\hline \multicolumn{4}{|l|}{ Clinical details } \\
\hline White cell count $\left(\times 10^{3} / \mathrm{mm}^{3}\right)$ & $10(8-13.6)$ & $12(10.5-15.5)$ & 0.029 \\
\hline Red cells in stoolb & I & 1 & 0.056 \\
\hline White cells in stoolb & 3 & 3 & 0.173 \\
\hline Mucoid duration (hrs) & $36(24-53.5)$ & $25(18-48)$ & 0.054 \\
\hline Diarrhea duration (hrs) & $48(39-72)$ & $48(27-72)$ & 0.088 \\
\hline \multicolumn{4}{|l|}{ Duration of illness } \\
\hline Hospital stay (days) & $5(4-5)$ & $4(3-5)$ & 0.276 \\
\hline Disease duration (days) ${ }^{c}$ & $7(6-8)$ & $5(4-7)$ & 0.009 \\
\hline
\end{tabular}

a $p$ Values calculated using either the Chi-square test or the Kruskal-Wallis test

b Cells in Stool assessed as described in methods

c Disease duration calculated by addition of history of disease and stay in hospital

$d$ Interquartile range values in brackets unless stated

serotypes, this approach may aid in tackling the global burden of shigellosis. The transition of dominant Shigella species in southern Vietnam has occurred on a background of economic development and may predict a continuing cycle in other areas under going similar rapid economic changes.

\section{Conclusions}

What we are unable to specifically ascertain from this study is the overall incidence and greater epidemiological picture of shigellosis in this setting. On the basis of these data a thorough epidemiological assessment of burden is warranted to calculate the financial and health implications of any potential future routine vaccination against shigellosis that may become available. However, here we have shown a significant transition in Shigella species and antimicrobial resistance dominance overtime and a concurrent change in the clinical disease presentation.

\section{Competing interests}

The authors declare that they have no competing interests.

\section{Authors' contributions}

NTKN, TVTN, PHD, JIC, NVMH, TTTN, PVM, CTT, PVBB and TSD performed the microbiological culturing, sensitivity testing and serotyping. MFB, PVTM provided critical analysis related to this work. HV, CP, LTP, MNL, BLM, VTCA, PVBB, HTL, MTC, NTHT, NVVC and JF conducted the clinical work providing the data for analysis. HV, JF, MFB and SB conceived the study, analyzed and interpreted the data and prepared the manuscript. All authors have read and approved the final version of this manuscript.

\section{Acknowledgements}

This work was supported by The Wellcome Trust, Euston Road, London, United Kingdom. MFB is supported by the Medical Research Council (grant G06007 I8). SB is supported by an Oxford University OAK fellowship. 


\section{References}

I. Miller MA, Sentz J, Rabaa MA, Mintz ED: Global epidemiology of infections due to Shigella, Salmonella serotype Typhi, and enterotoxigenic Escherichia coli. Epidemiol Infect 2008, I36(4):433-435.

2. Ram PK, Crump JA, Gupta SK, Miller MA, Mintz ED: Part II. Analysis of data gaps pertaining to Shigella infections in low and medium human development index countries, I 984-2005. Epidemiol Infect 2008, I 36(5):577-603.

3. Kotloff KL, Winickoff JP, Ivanoff B, Clemens JD, Swerdlow DL, Sansonetti PJ, Adak GK, Levine MM: Global burden of Shigella infections: implications for vaccine development and implementation of control strategies. Bull World Health Organ 1999, 77(8):65I-666.

4. Parry CM, Hien TT, Dougan G, White NJ, Farrar JJ: Typhoid fever. N Engl J Med 2002, 347(22): I770- I782.

5. Gupta A, Polyak CS, Bishop RD, Sobel J, Mintz ED: Laboratory-confirmed shigellosis in the United States, 1989-2002: epidemiologic trends and patterns. Clin Infect Dis 2004 38(10): I372-I377.

6. Mates A, Eyny D, Philo S: Antimicrobial resistance trends in Shigella serogroups isolated in Israel, 1990-1995. Eur J Clin Microbiol Infect Dis 2000, I 9(2): I08-III.

7. Clemens J, Kotloff K, Bradford K: Generic protocol to estimate the burden of Shigella diarrhoea and dysenteric mortality. The World Health Organization, Department of Vaccines and Biologicals 1999.

8. Niebuhr K, Sansonetti PJ: Invasion of epithelial cells by bacterial pathogens the paradigm of Shigella. Subcell Biochem 2000, 33:25I-287.

9. Philpott DJ, Edgeworth JD, Sansonetti PJ: The pathogenesis of Shigella flexneri infection: lessons from in vitro and in vivo studies. Philos Trans R Soc Lond B Biol Sci 2000, 355(I397):575-586.

10. von Seidlein L, Kim DR, Ali M, Lee H, Wang X, Thiem VD, Canh DG, Chaicumpa W, Agtini MD, Hossain A, et al.: A multicentre study of Shigella diarrhoea in six Asian countries: disease burden, clinical manifestations, and microbiology. PLoS Med 2006 , 3(9): e353.

II. Wirth T, Falush D, Lan R, Colles F, Mensa P, Wieler LH, Karch H, Reeves PR, Maiden MC, Ochman $H$, et al.: Sex and virulence in Escherichia coli: an evolutionary perspective. Mol Microbiol 2006, 60(5): I|36-|| $5 \mid$.

12. Farshad S, Sheikhi R, Japoni A, Basiri E, Alborzi A: Characterization of Shigella strains in Iran by plasmid profile analysis and PCR amplification of ipa genes. J Clin Microbiol 2006, 44(8):2879-2883.

13. Maurelli AT, Fernandez RE, Bloch CA, Rode CK, Fasano A: "Black holes" and bacterial pathogenicity: a large genomic deletion that enhances the virulence of Shigella spp. and enteroinvasive Escherichia coli. Proc Natl Acad Sci USA 1998 95(7):3943-3948.

14. Nie H, Yang F, Zhang X, Yang J, Chen L, Wang J, Xiong Z, Peng J, Sun $\mathrm{L}$, Dong J, et al.: Complete genome sequence of Shigella flexneri $\mathbf{5 b}$ and comparison with Shigella flexneri $2 a$. BMC Genomics 2006, 7:173.

15. Chompook P, Samosornsuk S, von Seidlein L, Jitsanguansuk S, Sirima N, Sudjai S, Mangjit P, Kim DR, Wheeler JG, Todd J, et al.: Estimating the burden of shigellosis in Thailand: 36-month populationbased surveillance study. Bull World Health Organ 2005, 83(10):739-746.

16. Vinh H, Wain J, Chinh MT, Tam CT, Trang PT, Nga D, Echeverria P, Diep TS, White NJ, Parry CM: Treatment of bacillary dysentery in Vietnamese children: two doses of ofloxacin versus 5-days nalidixic acid. Trans $R$ Soc Trop Med Hyg 2000, 94(3):323-326.

17. CLSI: Performance Standards For Antimicrobial Susceptibility Testing; Seventeenth Informational Supplement. 2007, 27( I):

18. Agtini MD, Soeharno R, Lesmana M, Punjabi NH, Simanjuntak C, Wangsasaputra F, Nurdin D, Pulungsih SP, Rofiq A, Santoso H, et al.: The burden of diarrhoea, shigellosis, and cholera in North Jakarta, Indonesia: findings from $\mathbf{2 4}$ months surveillance. BMC Infect Dis 2005, 5:89.

19. Wang XY, Du L, Von Seidlein L, Xu ZY, Zhang YL, Hao ZY, Han OP, Ma JC, Lee HJ, Ali M, et al.: Occurrence of shigellosis in the young and elderly in rural China: results of a I2-month population-based surveillance study. Am J Trop Med Hyg 2005, 73(2):416-422.
20. Hien BT, Trang do T, Scheutz F, Cam PD, Molbak K, Dalsgaard A: Diarrhoeagenic Escherichia coli and other causes of childhood diarrhoea: a case-control study in children living in a wastewater-use area in Hanoi, Vietnam. J Med Microbiol 2007, 56(Pt 8): 1086-1096.

21. Kim DR, Ali M, Thiem VD, Park JK, von Seidlein L, Clemens J: Geographic analysis of shigellosis in Vietnam. Health Place 2008 , I4(4):755-767.

22. Vinh H, Baker S, Campbell J, Hoang NV, Loan HT, Chinh MT, Anh VT, Diep TS, Phuong LT, Schultsz C, et al.: Rapid emergence of third generation cephalosporin resistant Shigella spp. in Southern Vietnam. I Med Microbiol 2009, 58(Pt 2):28I-283.

23. Guidelines for the control of shigellosis, including epidemics due to Shigella dysenteriae type I, from the World Health Organization workshop at the Centre for Health and Population Research Dhaka, Bangladesh, I6-I 8 February 2004 [http://whqlibdoc.who.int/publications/2005/924I592330.pdf]

24. Coster TS, Hoge CW, VanDeVerg LL, Hartman AB, Oaks EV Venkatesan MM, Cohen D, Robin G, Fontaine-Thompson A, Sansonetti PJ, et al:: Vaccination against shigellosis with attenuated Shigella flexneri $\mathbf{2 a}$ strain SC602. Infect Immun 1999, 67(7):3437-3443.

25. Katz DE, Coster TS, Wolf MK, Trespalacios FC, Cohen D, Robins G, Hartman AB, Venkatesan MM, Taylor DN, Hale TL: Two studies evaluating the safety and immunogenicity of a live, attenuated Shigella flexneri 2 a vaccine (SC602) and excretion of vaccine organisms in North American volunteers. Infect Immun 2004, 72(2):923-930.

26. Kotloff KL, Losonsky GA, Nataro JP, Wasserman SS, Hale TL, Taylor DN, Newland JW, Sadoff JC, Formal SB, Levine MM: Evaluation of the safety, immunogenicity, and efficacy in healthy adults of four doses of live oral hybrid Escherichia coli-Shigella flexneri $2 a$ vaccine strain EcSf2a-2. Vaccine 1995, I3(5):495-502.

27. Kotloff KL, Noriega FR, Samandari T, Sztein MB, Losonsky GA, Nataro JP, Picking WD, Barry EM, Levine MM: Shigella flexneri 2a strain CVD with specific deletions in virG, sen, set, and guaBA, is highly attenuated in humans. Infect Immun I207, 68(3): 1034-1039.

28. Dutta S, Rajendran K, Roy S, Chatterjee A, Dutta P, Nair GB, Bhattacharya SK, Yoshida SI: Shifting serotypes, plasmid profile analysis and antimicrobial resistance pattern of shigellae strains isolated from Kolkata, India during 1995-2000. Epidemiol Infect 2002, I 29(2):235-243.

29. Kotloff KL, Nataro JP, Losonsky GA, Wasserman SS, Hale TL, Taylor DN, Sadoff JC, Levine MM: A modified Shigella volunteer challenge model in which the inoculum is administered with bicarbonate buffer: clinical experience and implications for Shigella infectivity. Vaccine 1995, I3( I 6): | 488- 4994.

30. Ferreccio C, Prado V, Ojeda A, Cayyazo M, Abrego P, Guers L, Levine $M M$ : Epidemiologic patterns of acute diarrhea and endemic Shigella infections in children in a poor periurban setting in Santiago, Chile. Am J Epidemiol I991, I34(6):6|4-627.

\section{Pre-publication history}

The pre-publication history for this paper can be accessed here:

\section{http://www.biomedcentral.com/1471-2334/9/204/pre} pub 